\title{
Energy-efficient clustering routing algorithm for heterogeneous wireless sensor networks
}

\author{
Siqing Li \\ School of Internet of Things (IoT) Engineering, Jiangnan University, Wuxi, 214122, China \\ lisiqing885@163.com
}

\begin{abstract}
Energy heterogeneity is ubiquitous in wireless sensor networks. Because of nodes' initial energy of multi-level energy heterogeneity in wireless sensor networks distributing within a certain scope randomly, this paper proposed an Energy-efficient clustering routing algorithm which is suit to the heterogeneous environments in wireless sensor networks to economize the nodes' energy exploiting the energy heterogeneity and to extend the stability period of the network. The nodes of the cluster select the cluster head based on the current residual energy and the distance to the cluster centroid, and then accomplish the collection of data and the two-hop data transmission. The simulations indicate that the proposed algorithm balances the energy consumption among sensor nodes much better and prolongs the WSNs life time efficiently.

Index Terms - Wireless sensor network, energy heterogeneity, energy efficiency, clustering routing algorithm.
\end{abstract}

\section{Introduction}

Wireless sensor networks (WSNs) consist of a large number of low-power multifunctional sensor nodes with sensing, limited computation and wireless communications capabilities. Recent advances in sensor technology have enabled the development of small, low-cost and low-power sensors. In WSNs, sensors are densely deployed so that it can be applicable to a variety of fields that include surveillance, military, national security, and chemical or biological detection [1][2][3].

According to the initial energy, sensing capability, communication capacity and computing capacity, sensor node is divided into different categories. In the homogeneous sensor networks, all sensor nodes in them are the same type while the heterogeneous wireless sensor networks are composed of a variety of different types of sensor nodes. In the heterogeneous sensor networks, heterogeneous characteristics of the energy is universal, because different types of sensor nodes are configured with different initial energy, even if the nodes with the same type can also be configured with different initial energy. The multi-level heterogeneous sensor networks refer to that the energy of node presents a plurality of energy levels or randomly distributes within a certain range.

Hierarchical or cluster-based routing, are well-known techniques with special advantages related to scalability and efficient communications. As such, the concept of hierarchical routing is also utilized to perform energy-efficient routing in WSNs[4]. In a hierarchical architecture, higher energy nodes can be used to process and send the information while low energy nodes can be perform the sensing in the proximity of the target. Some of routing protocols in this group are: LEACH [5], LEACH-C [6], HEED [7] and DEEC [8].

LEACH (Low-Energy Adaptive Clustering Hierarchy) [5] is perhaps the first cluster based routing protocol for wireless sensor networks. It assumes that a unique base station exists outside the sensor networks and all the sensor nodes can communicate with this base station directly. In order to save energy, LEACH only chooses a fraction $\mathrm{p}$ of all sensor nodes to serve as cluster heads. The rest nodes join the proper clusters according to the signal strength from cluster heads, and its operation is divided into rounds. In each rounds, after cluster formation phase, the cluster heads aggregated data to the base station by single hop communication.

In Ref. [6], a new cluster-based routing protocol was proposed in order to distribute cluster heads evenly over the network and reduce energy dissipation. During the initial stage, each node has to send information about its current location and energy level to the base station. The sink finds clusters using the simulated annealing algorithm to solve the NP-hard problem of finding $C$ optimal clusters. This algorithm attempts to minimize the amount of energy for the ordinary nodes to transmit their data to the cluster head.

HEED [7] is a distributed clustering algorithm, which selects the cluster-heads stochastically. And HEED selects cluster heads based on the main parameters of nodes' residual energy, sub-parameters of the current node and the proximity of neighbors, which improves energy utilization of nodes and prolongs the network lifetime.

The heterogeneity of nodes in terms of their energy is considered in DEEC [8], which is designed for heterogeneous networks. In DEEC the cluster-heads are selected by a probability based on the ratio between the residual energy of each node and the average energy of the network.

In this paper, we propose and evaluate a new energyefficient clustering routing algorithm for heterogeneous wireless sensor networks, which is called EECRA. First, we use the fuzzy c-means (FCM) algorithm [9] to divide the whole network into c optimal cluster, and then select the cluster head according to the residual energy of node and the distance of node to the cluster center. The proposed algorithm guarantees that the node with higher energy and closer to the cluster center will be selected as the cluster head. And it balances the energy consumption of network effectively and extends the lifetime of network.

The rest of this paper is organized as follows. In section 2, we define the heterogeneous model and the optimal number of 
cluster head. Simulation results are presented in section 3 to evaluate the effectiveness of our design. Section 4 concludes the paper.

\section{System Model}

A. Heterogeneous network model

For our proposed model, We assume that $\mathrm{N}$ sensor nodes are distributed randomly in a $\mathrm{M} * \mathrm{M}$ two-dimensional square area $\mathrm{A}$, and periodically collect information on surroundings, as shown in Figure 1. A few reasonable assumptions of the network model as follows:

1) No mobility of sensor nodes.

2) The base station is fixed at a far distance from the sensor nodes.

3) The sensor nodes are heterogeneous and the energy can't recharge.

4) All nodes know themselves position information by GPS or other ranging location systems.

For multi-level heterogeneous networks, initial energy of sensor nodes is randomly distributed over the close set $\left[E_{0}, E_{0}\left(1+\alpha_{\max }\right)\right]$, where $E_{0}$ is the lower bound and $E_{0}\left(1+\alpha_{\max }\right)$ is the higher bound. The total initial energy of the multi-level heterogeneous networks is given by:

$$
E_{\text {total }}=\sum_{i=1}^{N} E_{0}\left(1+a_{i}\right)
$$

Where $a_{i}$ denotes the times energy more than $E_{0}$.

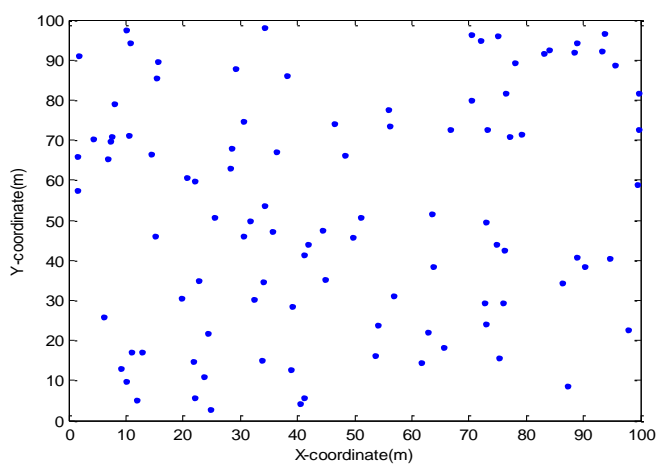

Fig. 1 A wireless sensor networks of 100 nodes uniformly deployed in a square area of side length $\mathrm{M}=100 \mathrm{~m}$

\section{B. Radio energy dissipation model}

In order to predict the performance of our proposed model, we use a simple radio hardware energy dissipation model shown in Ref. [6]. Both the free space and the multi-path fading channel models were used in the model according to the distance between the transmitter and receiver, that is to say $d^{2}$ (free space power loss) and $d^{4}$ (multi-path fading). The energy dissipation for transmission of $k$-bit message over distance $d$ is equal to Eq. (2), including the energy dissipated by the transmitter to run the radio electronics and the power amplifier.

$$
E_{T x}(k, d)=\left\{\begin{array}{l}
k E_{\text {elec }}+k \varepsilon_{f s} d^{2}, d<d_{0} \\
k E_{\text {elec }}+k \varepsilon_{\text {mp }} d^{4}, d \geq d_{0}
\end{array}\right.
$$

Where $E_{\text {elec }}$ is the electronics energy. $\varepsilon_{f s}$ and $\varepsilon_{\text {np }}$ are radio amplifier energies in different modes. And the threshold $d_{0}$ is: $d_{0}=\sqrt{\varepsilon_{f s} / \varepsilon_{n p}}$.

\section{Cluster head selection}

We assume that the $\mathrm{N}$ nodes are distributed uniformly in an $\mathrm{M} * \mathrm{M}$ region. Each non-cluster-head send $k$-bits data to the cluster-head per round. Thus the total energy dissipated in the network during a round is equal to:

$$
E_{\text {round }}=k\left(2 N E_{\text {elec }}+N E_{D A}+c \varepsilon_{\text {mp }} d_{\text {toBS }}^{4}+N \varepsilon_{\text {Is }} d_{\text {toOH }}^{2}\right)
$$

where $k$ is the number of clusters, $E_{D A}$ is the data aggregation cost expended in the cluster-heads, $d_{\text {tols }}$ is the average distance between the cluster-head and the base station, and $d_{\text {toOI }}$ is the average distance between the cluster members and the cluster-head. Assuming that the nodes are uniformly distributed, we can get:

$$
d_{\text {toOH }}=M / \sqrt{2 \pi c}, \quad d_{\text {toBS }}=0.765(M / 2)
$$

By setting the derivative of $E_{\text {round }}$ with respect to c to zero, we have the optimal number of cluster heads as Eq. (5) [10]:

$$
c_{\text {opt }}=\sqrt{N / 2 \pi} \sqrt{\varepsilon_{f s} / \varepsilon_{\text {mp }}} M / d_{\text {toBS }}^{2}
$$

\section{Eecra Protocol}

In this section, we proposed the Energy-efficient clustering routing algorithm for heterogeneous wireless sensor networks (EECRA). The proposed algorithm consists of two parts: First, we use the FCM algorithm divided the wireless sensor networks into several parts based on the optimal number of cluster heads $c_{o p t}$. Second, EECRA selects cluster head based on the residual energy of node and the distance from node to the cluster centroid. Compared with other algorithms, the proposed algorithm can effectively reduce the uneven distribution of cluster head space, while the energy consumption of network is more balanced.

\section{A. FCM clustering algorithm}

The FCM algorithm is based on the traditional K-means algorithm and the fuzzy set theory, which is a soft fuzzy classification method. The fundamental idea: First, the sensor nodes are processed into the $\mathrm{c}$ parts (where $\mathrm{c}$ is determined by the user), each part is given a cluster centroid (the cluster centroid tends to at a central location of each category). The FCM corrected the cluster centroid to the "right" position iteratively. The iteration based on minimizing an objective function (objective function is the distance from nodes to the corresponding cluster centroid) until the distance of nodes of each class to the corresponding cluster centroid weighted least.

FCM algorithm selects the cluster head and clustering calculation as follows: 
1) Fix the number of cluster $C$. Select randomly c nodes as initial centroids $V_{0}$ and then form partitions of all others nodes around these centroids to obtain the initial partition matrix $\mathrm{U}_{0}$ and membership values $u_{i j}$.

2) Computation of membership values $u_{i j}$

$$
u_{i j}=\left(\sum_{k=1}^{c}\left(\left\|X_{j}-V_{i}\right\| /\left\|X_{j}-V_{k}\right\|\right)^{2 /(m-1)}\right)^{-1}
$$

3) Computation of centroids $v_{i}$ :

$$
V_{i}=\sum_{j=1}^{N} u_{i j}{ }^{m} x_{j} / \sum_{j=1}^{N} u_{i j}{ }^{m}
$$

4) Compare $v_{i}(k)$ and $v_{i}(k+1)$ in a convenient matrix norm, if $e=\sum_{i=1}^{c}\left\|V_{i}(k+1)-V_{i}(k)\right\|^{2}$ and $\mathrm{e}<\zeta$, stop, otherwise, set $\mathrm{k}=\mathrm{k}+1$, go to step 2).

According to the optimal system number cluster heads $C$, the whole network is divided into $C$ clusters area by FCM clustering algorithm. Each node belongs to one cluster region. In the whole lifetime of the network, the divided regional structure of the cluster will be fixed, that is to say, once each node is determined to belong to the specified cluster, it will never change until the node fails. As shown in Figure 2.

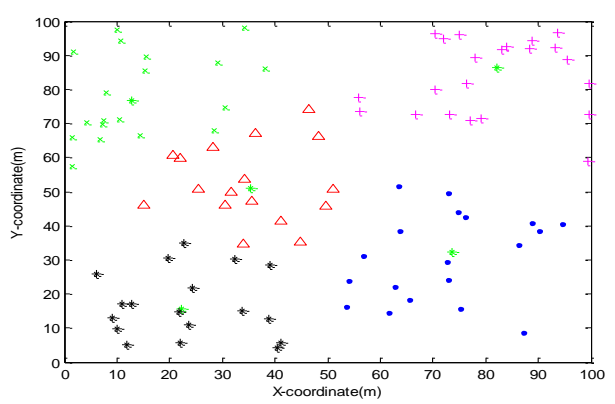

Fig. 3 The initial clustering centers

\section{B. Cluster head selection}

Each node is assigned a degree of belonging to cluster head rather than completely being a member of just one cluster. Therefore, the node close to the boundary of a cluster may become members of the neighbor clusters.

After the cluster is created, the non-cluster head nodes send data toward the base station through the cluster heads. The process of selecting clusters is repeated every round of exchanging data among sensor nodes. Only at the first stage, the cluster head of each cluster is chosen by the base station, after that the current cluster head makes decision of selecting which node will become the cluster head at the next round. In the selection of cluster head, we first introduced the two parameters, $E_{\text {residual }}(i)$ and distance $(i)$, which denotes the remaining energy of nodes and the distance from node to the cluster centroid, respectively. During the transmission from the sensor nodes to cluster head, residual energy of each nodes are attached to the data packet, the information assists the cluster head to choose the node with the highest residual energy and nearest to the cluster centroid to be cluster head at the next round. As shown in Equation (8):

$$
\operatorname{cost}(\mathrm{i})=\alpha *(1 / \operatorname{distanc}(\mathrm{i}))+\beta * \mathrm{E}_{\text {residual }}(\mathrm{i})
$$

Where $\alpha$ and $\beta$, respectively, which means that the weight of two parameter. At the same time, $\alpha$ and $\beta$ were taken the experience value 0.3 and 0.7 , and $\alpha+\beta=1$.

Based on the number of alive nodes within the cluster, the new cluster head creates a TDMA schedule to allocate the time when cluster members can transmit.

\section{Data transmission}

Once the cluster heads are selected and the transmission scheduled is made, the sensor nodes start to transmit data to the cluster heads. The radio of each non-cluster head node can be turned off until the node's allocated transmission time, thus that minimizes the energy dissipation in these nodes. Simultaneously, the transmission power of non-cluster head nodes is optimized because of the minimum spatial distance to the cluster heads achieved by FCM algorithm. The clusterhead node must keep its receiver on to receive all the data from the nodes in the cluster. When all the data has been received, the cluster head node performs signal processing functions to compress the data into a single signal. For example, if the data are audio or seismic signals, the clusterhead node can beamform the individual signals to generate a composite signal. This composite signal is sent to the base station.

\section{Simulation Results}

In this section, we evaluate the performance of the EECRA protocol. We have considered first order radio model simulation to LEACH and the simulation parameters for our model are mentioned in Table 1. To validate the performance of EECRA, we simulate a heterogeneous clustering WSN in a field with dimensions $100 \mathrm{~m}^{*} 100 \mathrm{~m}$. The total number of sensor nodes $n=100$. Sink node is located at $(0,0)$.

TABLE 1 Transmission parameters value

\begin{tabular}{|l|l|}
\hline Parameter & Value \\
\hline Data packet size $(\mathrm{b})$ & 4000 \\
\hline$E_{\text {elec }} \mathrm{nJ} / \mathrm{b}$ & 50 \\
\hline$k \varepsilon_{f s} \mathrm{p} /\left(\mathrm{b} \bullet \mathrm{m}^{2}\right)$ & 10 \\
\hline$k \varepsilon_{m p} \mathrm{p} /\left(\mathrm{b} \bullet \mathrm{m}^{4}\right)$ & 0.0013 \\
\hline$E_{D A} \mathrm{~nJ} /(\mathrm{b} \cdot$ signal $)$ & 5 \\
\hline $\mathrm{C}$ & 5 \\
\hline Threshold distance $d_{0}(\mathrm{~m})$ & 87.7 \\
\hline
\end{tabular}

In Fig.3, the number of alive nodes over the operating time of the network by using different protocol is compared. In our work, the performance of LEACH and EECRA are studied. The different duration of time up to the first dead node by applying different protocols is given in Table 2 . It is obviously seen that the lifetime of the network with $100 \%$ nodes alive using EECRA protocol is much longer than that when LEACH is employed. 
TABLE 2 Ten times the duration of time up to the first node dies in the network

\begin{tabular}{|c|c|c|}
\hline Times & LEACH & EECRA \\
\hline 1 & 800 & 1300 \\
\hline 2 & 812 & 1350 \\
\hline 3 & 780 & 1301 \\
\hline 4 & 795 & 1290 \\
\hline 5 & 805 & 1289 \\
\hline 6 & 823 & 1310 \\
\hline 7 & 779 & 1333 \\
\hline 8 & 803 & 1290 \\
\hline 9 & 810 & 1293 \\
\hline 10 & 778 & 1320 \\
\hline
\end{tabular}

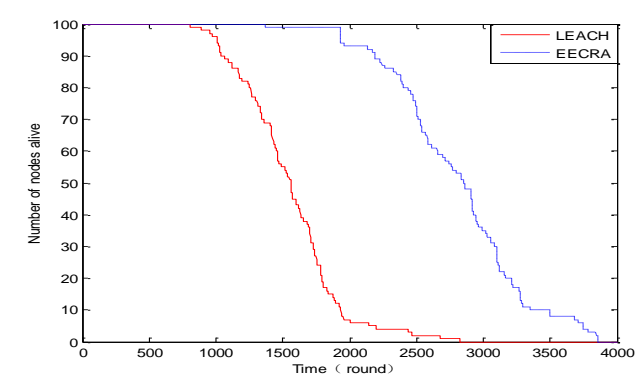

Fig.3 Number of nodes alive over the time with different cluster based protocols

In Fig.4, we see the average energy dissipation in the network per round. It shows that the energy dissipation of EECRA is less than that of LEACH. Moreover, the lifetime of EECRA is longer than that of LEACH. Now EECRA takes full advantages of heterogeneity, and the stable region increases ssignificantly in comparison with that of LEACH. This is because under EECRA, the node with higher residual energy and closer to the cluster center will be the cluster head.

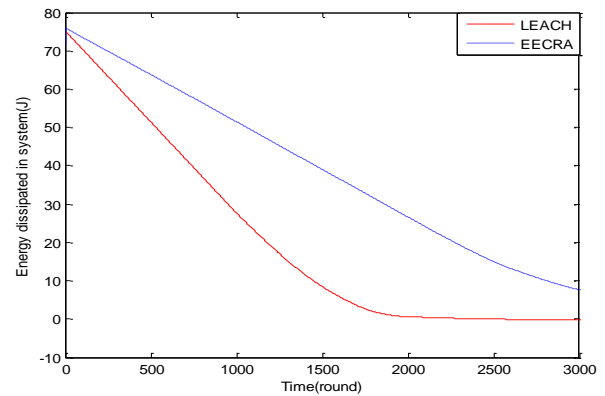

Fig.4 Comparison of average energy dissipation

\section{Conclusion}

In this paper, we present a energy-efficient clustering routing algorithm for heterogeneous wireless sensor networks, EECRA. This protocol uses FCM algorithm to create cluster structure in order to minimize the spatial distance among the sensor nodes and thus a better cluster formation is obtained. The node with the highest residual energy and nearest to the cluster center to be the cluster head. Our simulation results show that by the EECRA algorithm the power consumption is reduced and the life time of the network is extended significantly when compared with LEACH.

\section{VI . Acknowledgment}

We thank our group members for their help during the development of this project and their invaluable comments on many rounds of earlier drafts. Special thanks to Mr. Huang for his verification on the simulation results.

\section{References}

[1] Krishnamachari Bhaskar. "Networking Wireless Sensors," London: Cambridge University Press, 2005

[2] I.F.Akyildiz, W.Su, Y.S.karasubramaniam, et al. "A Survey on Sensor Networks," IEEE Communications Magazine, 2002, vol.40, no.8, pp.102-114

[3] R. Szewczyk, E. Osterweil, J. Polastre, et al. Habitat monitoring with sensor networks. Communications of the ACM, 2004, vol.47, no.6, pp. $34-40$

[4] JN. Al-Karaki, AE. Kamal. "Routing techniques in wireless sensor network: a survey," IEEE Wireless communication, 2004, vol.11, no.6, pp.6-28

[5] WR. Heinzelman, A. Chandrakasan, H. Balakrishnan. "Energy-Efficient Communication Protocol for Wireless Microsensor Networks," in Proc. the 33rd Annual Hawaii International Conference on System Sciences, 2000, vol.2, pp. 1-10

[6] W.R.Heinzelman, A.P. Chandrakasan and H. Balakrishnan. "An Application-Specific Protocol Architecture for Wireless Microsensor Networks," IEEE Trans on Wireless Communication, 2002, vol.1, no.4, pp. $660-670$

[7] O. Younis, S. Fahmy. "HEED: A hybrid, energy-efficient, distributed clustering approach for ad hoc sensor networks," IEEE Trans. on Mobile Computing, 2004, vol.3, no.4, pp.660-669.

[8] L. Qing, Q. Zhu, M. Wang. "Design of a distributed energy-efficient clustering algorithmfor heterogeneous wireless sensor networks," Computer Communications, 2006, vol.29, pp.2230-2237.

[9] R. Duda, P. Hart and D. Stork. "Pattern Classification, Second Edition," New Jersey: John-Wiley, 2000

[10] Dilip Kumar, Trilok C. Aseri, R.B. Patel. "EEHC: Energy efficient heterogeneous clustered scheme for wireless sensor networks," Computer Communications, 2009, vol.32, no.4, pp.662-667 